\title{
Socioeconomic status, non-communicable disease risk factors, and walking speed in older adults: multi-cohort population based study
}

\author{
Silvia Stringhini, ${ }^{1}$ Cristian Carmeli, ${ }^{1}$ Markus Jokela, ${ }^{2}$ Mauricio Avendaño, ${ }^{3,4}$ Cathal McCrory, ${ }^{5}$ \\ Angelo d'Errico, ${ }^{6}$ Murielle Bochud, ${ }^{1}$ Henrique Barros, ${ }^{7,8}$ Giuseppe Costa, ${ }^{6}$ Marc Chadeau-Hyam, ${ }^{9}$ \\ Cyrille Delpierre, ${ }^{10}$ Martina Gandini, ${ }^{6}$ Silvia Fraga, ${ }^{7}$ Marcel Goldberg, ${ }^{11}$ Graham G Giles, ${ }^{12}$ \\ Camille Lassale, ${ }^{13}$ Rose Anne Kenny, ${ }^{5}$ Michelle Kelly-Irving, ${ }^{10}$ Fred Paccaud, ${ }^{1}$ Richard Layte, ${ }^{14}$ \\ Peter Muennig, ${ }^{15}$ Michael G Marmot, ${ }^{13}$ Ana Isabel Ribeiro, ${ }^{7}$ Gianluca Severi, ${ }^{12,16,17}$ \\ Andrew Steptoe, ${ }^{13}$ Martin J Shipley, ${ }^{13}$ Marie Zins, ${ }^{11}$ Johan P Mackenbach, ${ }^{18}$ Paolo Vineis, ${ }^{9}$ \\ Mika Kivimäki, ${ }^{13,19}$ for the LIFEPATH Consortium
}

For numbered affiliations see end of article.

Correspondence to: S Stringhini silvia.stringhini@chuv.ch

Additional material is published online only. To view please visit the journal online.

Cite this as: $B M J$ 2018;360:k1046 http://dx.doi.org/10.1136/bmj.k1046

Accepted: 20 February 2018

\section{ABSTRACT}

OBJECTIVE

To assess the association of low socioeconomic status and risk factors for non-communicable diseases (diabetes, high alcohol intake, high blood pressure, obesity, physical inactivity, smoking) with loss of physical functioning at older ages.

\section{DESIGN}

Multi-cohort population based study.

SETTING

37 cohort studies from 24 countries in Europe, the United States, Latin America, Africa, and Asia, 1990-2017.

PARTICIPANTS

109107 men and women aged 45-90 years.

MAIN OUTCOME MEASURE

Physical functioning assessed using the walking speed test, a valid index of overall functional capacity. Years of functioning lost was computed as a metric to quantify the difference in walking speed between those exposed and unexposed to low socioeconomic status and risk factors.

\section{WHAT IS ALREADY KNOWN ON THIS TOPIC}

Years of life lost due to unfavourable socioeconomic circumstances and noncommunicable disease risk factors have been estimated, but the extent to which such factors affect physical functioning is unknown

In addition to prevention of age related morbidity and premature mortality, minimising the time that people spend living with disability and dependence is a major public health challenge

Walking speed, an indicator of physical functioning, declines with age and is a predictor of survival, hospital admission, and cognitive decline

\section{WHAT THIS STUDY ADDS}

The independent association between socioeconomic status and walking speed in old age is comparable in strength and consistency (across sex and age groups) to those for leading non-communicable disease risk factors

Overall, 4 to 7 years of good physical functioning are lost due to poor socioeconomic circumstances at age 60 and the corresponding loss for other risk factors are 0.5 to 8 years

Physical functioning continues to decline as a function of socioeconomic status and unfavourable risk factors at least until age 85

\section{RESULTS}

According to mixed model estimations, men aged 60 and of low socioeconomic status had the same walking speed as men aged 66.6 of high socioeconomic status (years of functioning lost 6.6 years, $95 \%$ confidence interval 5.0 to 9.4$)$. The years of functioning lost for women were 4.6 (3.6 to 6.2). In men and women, respectively, 5.7 (4.4 to 8.1) and 5.4 (4.3 to 7.3) years of functioning were (3.9 to 7.0) and 7.5 (6.1 to 9.5) due to obesity, 2.3 (1.6 to 3.4) and 3.0 (2.3 to 4.0) due to hypertension, 5.6 (4.2 to 8.0) and 6.3 (4.9 to 8.4) due to diabetes, and 3.0 (2.2 to 4.3) and 0.7 (0.1 to 1.5$)$ due to tobacco use. In analyses restricted to high income countries, the number of years of functioning lost attributable to low socioeconomic status by age 60 was 8.0 ( 5.7 to 13.1 ) for men and 5.4 ( 4.0 to 8.0) for women, whereas in low and middle income countries it was $2.6(0.2$ to 6.8$)$ for men and 2.7 (1.0 to 5.5) for women. Within high income countries, the number of years of functioning lost attributable to low socioeconomic status by age 60 was greater in the United States than in Europe. Physical functioning continued to decline as a function of unfavourable risk factors between ages 60 and 85 . Years of functioning lost were greater than years of life lost due to low socioeconomic status and non-communicable disease risk factors.

\section{CONCLUSIONS}

The independent association between socioeconomic status and physical functioning in old age is comparable in strength and consistency with those for established non-communicable disease risk factors.

The results of this study suggest that tackling all these risk factors might substantially increase life years spent in good physical functioning.

\section{Introduction}

In the context of a rapidly aging world population, global health strategies have considered healthy aging as a public health priority. ${ }^{12}$ The World Health Organization Global Action Plan for the Prevention and Control of Non-Communicable Diseases, for example, has set member states a goal by 2025 to reduce premature mortality from chronic diseases lost by age 60 due to insufficient physical activity, 5.1 
by $25 \% .^{3}$ This is to be achieved by targeting high alcohol consumption, insufficient physical activity, tobacco use, high blood pressure, excess salt intake, diabetes, and obesity. Recent studies suggest that targeting adverse socioeconomic circumstances or low socioeconomic status in addition to these risk factors might lead to additional gains in longevity. ${ }^{45}$ Socioeconomic status is a sociological construct referring to an individual's relative position in the social hierarchy, as measured by indicators such as occupational group, educational attainment, level of income and wealth, and place of residence.

In addition to prevention of age related morbidity and premature mortality, minimising the time that people spend living with disability and dependence is a major public health challenge. Although several investigations have found socioeconomic status and risk factors for non-communicable diseases to predict mobility and disability, ${ }^{6-18}$ whether socioeconomic status has a similar predictive value for functioning as established non-communicable disease risk factors has not yet been explored. Using national data across different contexts and regions to examine the consistency of the associations of socioeconomic status and non-communicable risk factors with physical functioning and mortality within a single analytic setting would be useful because of implications for priority setting of risk factors as well as planning health and social policies. We hypothesised that socioeconomic status is related to greater absolute differences in physical functioning at older ages than younger ages as is the case for established noncommunicable disease risk factors.

We compared the association between socioeconomic status and physical functioning with those between established non-communicable disease risk factors and physical functioning from early to advanced age. To obtain reliable and generalisable estimates, we pooled harmonised individual level data from 37 studies spanning 24 countries from Europe, the United States, Europe, Latin America, Africa, and Asia. We used walking speed as a measure of physical functioning because it is a simple and sensitive indicator of overall functional capacity. ${ }^{19}$ As walking is a complex task requiring energy, balance, movement control, and coordination of the musculoskeletal, nervous, respiratory, and cardiovascular systems, ${ }^{19}$ disturbance or deterioration in any of these modalities tends to affect the speed of walking. ${ }^{20} 21$ Furthermore, walking speed declines with age and predicts functional limitations, hospital admission, clinical and subclinical disease, comorbidities, frailty, admission to residential care, and mortality, ${ }^{19} 22-28$ even in old age. ${ }^{29}$

\section{Methods}

\section{Study populations}

The present study is part of an EC Horizon 2020 consortium, the LIFEPATH project. In the present analysis we included a total of 37 studies comprising 109107 men and women aged 45 to 90 years from
24 WHO member countries: UK, France, USA, Mexico, China, Ghana, India, Russia, South Africa, Costa Rica, Taiwan, Ireland, Austria, Belgium, Denmark, Germany, Greece, Israel, Italy, the Netherlands, Portugal, Spain, Sweden, and Switzerland. We chose the 45-90 age range as few data were available for younger or older age groups and the meaning of walking speed in the age group 30-40 years is unclear.

Data were collected between 1990 and 2017. All studies included data on socioeconomic status and walking speed. We excluded people who used walking aids because walking speed was used as a proxy measure of overall functioning, and the assessment is not reliable among participants using walking aids. The relevant local or national ethics committees approved each study, and participants gave informed consent to participate. Six datasets were part of the LIFEPATH Consortium (the GAZEL, ELSA, WHITEHALL II, CONSTANCES, TILDA, and EPIPORTO studies), six were part of the WHO Study on global AGEing and adult health (SAGE), 12 were from the Survey of Health, Ageing and Retirement in Europe (SHARE), four were from the InterUniversity Consortium for Political and Social Research (MIDUS, HEPESE, SEBAS, and NSHAP), one was from the Health and Retirement Study (HRS), two were from the Wisconsin Longitudinal Study (WLSG and WLSS), three were from the National Health and Nutrition Examination Survey (NHANES III, NHANES 1999, and NHANES 2001), two were from the Costa Rican Longevity and Health Ageing Study (CRELES Pre 1945 and CRELES RC), and one was from the Health and Ageing Study in Africa: A Longitudinal Study of an INDEPTH Community in South Africa (HAALSI). The appendix provides details of the study design, participants, and cohort descriptions.

\section{Risk factors}

In the LIFEPATH Consortium, we used a predefined harmonised definition of socioeconomic status, as described in previous papers. ${ }^{30} 31$ We used information on several social indicators available in the participating cohort studies-education, own and father's occupational class, and income. For adults, we assigned socioeconomic status to cohort members using information on the last known occupational title at study enrolment. To obtain a harmonised measure of occupational class across the study cohorts, we classified occupations according to the European socioeconomic classification, which is a classification based on the nature of employment relationships. ${ }^{32}$ We predefined and harmonised such data across the study cohorts before analyses. ${ }^{4}$ We categorised occupational class into high (higher professionals and managers, higher clerical, services, and sales workers (European socioeconomic class 1-3)), intermediate (small employers and self employed, farmers, lower supervisors and technicians, class 4-6), or low socioeconomic status (lower clerical, services, and sales workers, skilled workers, semiskilled and unskilled workers, class 7-9). ${ }^{33}$ Given the large proportion of participants $(>50 \%)$ with no formal 
occupation, in the case of HAALSI, we used a measure of wealth categorised in thirds.

Self reported smoking was categorised into current, former, and never. Alcohol consumption was measured in alcohol units weekly, and we categorised participants as non-drinkers ( 0 units/week), moderate drinkers (1-21 units/week for men, 1-14 units/week for women), or harmful drinkers ( $>21$ units/week for men, >14 units/week for women). Leisure physical activity was measured with different questions in each study, making it impossible to derive a comprehensive definition. As a result, we dichotomised leisure physical activity as sufficient or insufficient in each study using study specific thresholds (see supplementary appendix 2). Height and weight were measured using standard procedures; body mass index (BMI) was calculated as $\mathrm{kg} / \mathrm{m}^{2}$ and categorised as normal (18.5 to $<25$ ), overweight $(25$ to $<30)$, or obese $(\geq 30)$, with those who were underweight $(<18.5)$ excluded from the BMI analyses. Hypertension was defined as the presence of at least one of systolic blood pressure $\geq 140 \mathrm{~mm} \mathrm{Hg}$, diastolic blood pressure $\geq 90 \mathrm{~mm} \mathrm{Hg}$, current antihypertensive treatment, or self reported hypertension. Diabetes was defined as the presence of at least one of fasting glucose concentration $\geq 7 \mathrm{mmol} / \mathrm{L}$, post-load glucose concentration $>11.1$ $\mathrm{mmol} / \mathrm{L}$ at two hours, glycated haemoglobin A1c level $\geq 6.5 \%$, or self reported diabetes.

\section{Covariates}

We considered age, height, and year of birth (five year intervals) as potential confounders. Height is a correlate of walking speed, as longer legs are associated with higher walking speed (allometric dependence of walking speed). In addition, height is a marker of childhood health or disease, which can affect both walking speed in adulthood and functioning in old age. In supplementary models, we also controlled for ethnic origin and baseline health status.

\section{Walking speed}

In all cohorts, participants were instructed to walk at their usual pace. The setup differed among cohorts, (see supplementary table S1 for details). The distance walked varied from 8 feet (2.4 m) in ELSA, WHITEHALL II, NHANES III, and HEPESE to $15.24 \mathrm{~m}$ in MIDUS. Walking time was recorded with photoelectric devices for some cohorts and manually by an interviewer for others. In all studies, time walked was averaged across multiple trials and was then converted into an overall measure of speed, expressed as metres travelled per second ( $\mathrm{m} / \mathrm{s})$. Walking speed was assessed at the same time as participant risk factors.

\section{Mortality}

In a subset of 24 cohorts (table 1), 83783 participants were linked to national mortality registries that provided information about vital status. We set the baseline for mortality analysis at the same wave in which gait speed measures were obtained. Mean mortality follow-up ranged between 2.9 years (MIDUS) and 21.4 years (NHANES 3), with a mean of 8.1 years (SD 5.2 years) across cohorts.

\section{Statistical analysis}

Walking speed and age

We used a generalised additive mixed model (GAMM) $)^{34}$ to estimate walking speed, with age and height as fixed effect predictors and study as random effect at the intercept and age slope. GAMM is a semi-parametric model that uses a family of splines to obtain a smooth representation of the dependence of walking speed with age. The algorithm implemented in the R package gamm4 automatically selected the number of knots in the splines. ${ }^{35}$ We computed $95 \%$ confidence intervals from the uncertainty of the estimated smoothing function.

\section{Years of functioning lost}

We computed the number of years of functioning lost from the mixed model predictions of walking speed along with age. The mixed model of walking speed included a random effect of study at the intercept and age slope. Fixed effects included age, age ${ }^{2}$, height, year of birth, distances walked, the risk factor under study (minimally adjusted models) or all risk factors (mutually adjusted models), and an interaction term between age and the risk factor. The structure of the models was determined through likelihood ratio tests.

Confidence intervals for years of functioning lost were determined through 5000 bootstrap samples, applying a model based parametric bootstrap method. ${ }^{36}$ For all examined risk factors, we computed the years of functioning lost associated with exposure by predicting the chronological age of the unexposed group equivalent to the walking speed at age 60 (or 85 ) of the exposed group. Years of functioning lost was then obtained as (60 (or 85) age reference). This method allows years of functioning lost at a given age to be calculated retrospectively, as opposed to the classic years of life lost calculated prospectively.

\section{Years of life lost}

Years of life lost were used as a secondary outcome, and we compared this with years of functioning lost, our primary outcome. We calculated years of life lost as the difference between the areas under the survival curves (from age 60 to 85 ) of the population exposed to a given risk factor compared with the unexposed reference population. Survival curves were estimated using Kaplan-Meier adjusted curves, conditional on survival to age 60 years. We ran a shared frailty Cox model ${ }^{19}$ with age as time scale, stratified by the levels of the given risk factor and year of birth as covariate (for minimally adjusted models) or year of birth and the remaining risk factors as covariates (mutually adjusted models). The shared frailty variable was introduced to account for study effect. ${ }^{19}$

\section{Patient involvement}

No patients were involved in setting the research question or the outcome measures, nor were they 


\begin{tabular}{|c|c|c|c|c|c|c|c|}
\hline Study & $\begin{array}{l}\text { Baseline for } \\
\text { this study }\end{array}$ & Country & No of participants & $\begin{array}{l}\text { Mean (SD) age at } \\
\text { baseline (years) }\end{array}$ & $\begin{array}{l}\text { Walking } \\
\text { distance (m) }\end{array}$ & $\begin{array}{l}\text { Mean walking } \\
\text { speed }(\mathrm{m} / \mathrm{s})\end{array}$ & $\begin{array}{l}\text { Mean mortality } \\
\text { follow-up (years) }\end{array}$ \\
\hline GAZEL & 2010 & France & $1979 / 603$ & $64.5(2.8) / 61.7(4.0)$ & 3 & $1.18 / 1.15$ & $6.6 / 6.6$ \\
\hline ELSA & 2004-05 & UK, England & $2104 / 2545$ & $70.1(7.2) / 70.4(7.6)$ & 2.44 & $0.91 / 0.84$ & $7.3 / 7.6$ \\
\hline WHITEHALL II & $2002-04$ & UK, London & $4384 / 1809$ & $61.0(5.9) / 61.4(6.0)$ & 2.44 & $1.28 / 1.13$ & $9.1 / 9.2$ \\
\hline NHANES III & $1990-93$ & USA & $2277 / 2184$ & $71.6(8.0) / 71.9(8.1)$ & 2.44 & $0.75 / 0.70$ & $9.9 / 11.2$ \\
\hline NHANES 1999 & 1999 & USA & $882 / 801$ & $66.3(9.8) / 65.7(10.1)$ & 6.1 & $0.97 / 0.94$ & $9.8 / 10.4$ \\
\hline NHANES 2001 & 2001 & USA & $925 / 871$ & $65.3(10.2) / 65.5(10.3)$ & 6.1 & $1.03 / 1.0$ & $8.8 / 9.2$ \\
\hline HRS & 2006-09 & USA & $2685 / 3110$ & $72.4(6.3) / 72.0(6.5)$ & 5 & $1.58 / 1.48$ & $6.3 / 6.5$ \\
\hline MIDUS & 2004-05 & USA & $154 / 154$ & $52.6(6.7) / 52.2(6.2)$ & 15.24 & $1.12 / 1.08$ & $3.0 / 3.0$ \\
\hline WLSG & 2010-12 & USA, Wisconsin & $2519 / 2717$ & $71.3(0.9) / 71.2(0.9)$ & 2.5 & $1.01 / 0.95$ & $3.4 / 3.3$ \\
\hline WLSS & $2010-12$ & USA, Wisconsin & $1333 / 1465$ & $68.7(6.7) / 69.0(6.6)$ & 2.5 & $1.02 / 0.96$ & $3.3 / 3.3$ \\
\hline CONSTANCES & 2012 & France & $13593 / 14819$ & $57.9(7.1) / 57.4(7.0)$ & 3 & $1.29 / 1.25$ & None \\
\hline CRELES-RC & 2010 & Costa Rica & $1196 / 1766$ & $61.0(5.3) / 58.7(4.3)$ & 3 & $1.04 / 0.98$ & None \\
\hline CRELES Pre 1945 & 2005 & Costa Rica & $901 / 702$ & $73.6(7.6) / 72.4(8.0)$ & 3 & $0.69 / 0.62$ & $3.0 / 3.1$ \\
\hline HAALSI & 2014-15 & $\begin{array}{l}\text { South Africa, } \\
\text { Agincourt }\end{array}$ & $1691 / 1955$ & $63.4(10.6) / 62.5(10.8)$ & 2.5 & $0.63 / 0.58$ & None \\
\hline HEPESE & 1993-94 & $\begin{array}{l}\text { USA, Mexican } \\
\text { Americans }\end{array}$ & $966 / 723$ & $72.6(5.8) / 72.2(5.5)$ & 2.44 & $0.46 / 0.43$ & $5.4 / 5.9$ \\
\hline SEBAS & 2006 & Taiwan & $514 / 331$ & $66.2(9.5) / 63.3(8.8)$ & 3 & $0.86 / 0.80$ & None \\
\hline NSHAP & 2010-11 & USA & $1389 / 1626$ & $72.7(7.2) / 71.4(8.1)$ & 3 & $0.70 / 0.68$ & None \\
\hline SAGE China & 2008 & China & $4652 / 4786$ & $62.3(9.4) / 61.8(9.4)$ & 4 & $1.02 / 0.96$ & None \\
\hline SAGE Ghana & 2008 & Ghana & $2020 / 1804$ & $61.7(10.0) / 63.2(10.2)$ & 4 & $0.81 / 0.71$ & None \\
\hline SAGE India & 2008 & India & $3150 / 1661$ & $60.7(9.0) / 58.4(8.9)$ & 4 & $0.89 / 0.81$ & None \\
\hline SAGE Mexico & 2008 & Mexico & $502 / 370$ & $65.5(9.0) / 64.5(9.3)$ & 4 & $0.88 / 0.78$ & None \\
\hline SAGE Russia & 2008 & Russia & $884 / 1570$ & $61.4(9.1) / 63.3(9.9)$ & 4 & $0.77 / 0.70$ & None \\
\hline SAGE South Africa & 2008 & South Africa & $901 / 1159$ & $61.5(9.0) / 62.1(9.5)$ & 4 & $0.85 / 0.77$ & None \\
\hline SHARE Austria & 2004 & Austria & $42 / 48$ & $76.0(8.8) / 77.1(8.2)$ & 5 & $1.36 / 1.33$ & $6.8 / 7.1$ \\
\hline SHARE Belgium & 2004-05 & Belgium & $170 / 162$ & $78.6(6.3) / 77.6(7.1)$ & 5 & $1.47 / 1.26$ & $6.0 / 6.7$ \\
\hline SHARE Denmark & 2004 & Denmark & $84 / 105$ & $77.4(9.1) / 77.3(8.5)$ & 5 & $1.62 / 1.42$ & $6.7 / 7.4$ \\
\hline SHARE France & 2004-05 & France & $141 / 177$ & $77.6(7.1) / 78.3(7.3)$ & 5 & $1.33 / 1.20$ & $6.2 / 6.8$ \\
\hline SHARE Germany & 2004 & Germany & $91 / 100$ & $76.5(7.1) / 78.0(6.3)$ & 5 & $1.37 / 1.23$ & $5.9 / 6.3$ \\
\hline SHARE Greece & 2004-05 & Greece & $83 / 69$ & $78.7(6.8) / 76.1(8.8)$ & 5 & $1.14 / 1.07$ & $4.5 / 4.5$ \\
\hline SHARE Israel & $2005-06$ & Israel & $71 / 49$ & $78.5(5.8) / 76.8(7.6)$ & 5 & $1.45 / 1.27$ & $6.5 / 7.2$ \\
\hline SHARE Italy & 2004 & Italy & $85 / 76$ & $75.8(7.4) / 74.1(9.3)$ & 5 & $1.28 / 1.02$ & $7.1 / 7.5$ \\
\hline SHARE Netherlands & 2004 & Netherlands & $124 / 107$ & $77.7(7.7) / 76.5(8.5)$ & 5 & $1.53 / 1.46$ & $5.6 / 7.5$ \\
\hline SHARE Spain & 2004 & Spain & $118 / 115$ & $77.4(7.6) / 75.0(9.3)$ & 5 & $1.26 / 1.08$ & $7.2 / 7.6$ \\
\hline SHARE Sweden & 2004-05 & Sweden & $129 / 141$ & $79.5(5.7) / 79.5(5.5)$ & 5 & $1.57 / 1.43$ & $6.6 / 7.3$ \\
\hline SHARE Switzerland & 2004 & Switzerland & $43 / 56$ & $80.7(4.3) / 79.9(6.2)$ & 5 & $1.66 / 1.45$ & $5.6 / 7.1$ \\
\hline TILDA & 2009-11 & Ireland & $2149 / 2638$ & $62.0(8.4) / 60.5(8.4)$ & 4.88 & $1.38 / 1.36$ & None \\
\hline EPIPORTO & 2016-17 & Portugal & $324 / 478$ & $65.7(10.3) / 65.1(9.4)$ & 7.62 & $1.74 / 1.39$ & None \\
\hline
\end{tabular}

involved in developing plans for recruitment, design, or implementation of the study. No patients were asked to advise on interpretation or writing up of results. There are no plans to disseminate the results of the research to study participants or the relevant patient community.

\section{Results}

Thirty seven studies from 24 countries were included. Of 140092 participants with data on walking speed, we excluded 30985 (8912 were outside the studied age range (45-90 years), 18545 (14.1\%) lacked data on socioeconomic status, 1468 lacked data on one or more of the covariates, and 2060 (1.9\%) used a walking aid). This left 109107 participants for analysis (fig 1). Compared with those included in the analyses, the excluded participants were younger ( $58.1 \mathrm{v} 63.7$ years, this is because we set 45 years as the minimum age), of a lower socioeconomic status $(36.7 \% \mathrm{~V} 32.2 \%)$, and more likely to be women $(64.8 \% v 49.4 \%)$. They were also more likely to have a normal BMI (46.8\% v 41.0\%), abstain from alcohol (64.3\% v 42.9\%), be physically inactive (32.4\% $v 29.4 \%$ ), have hypertension (59.4\% $\mathrm{V}$ $55.2 \%)$, and smoke (22.1\% v15.2\%).
Of the 109107 participants, 14368 (13.2\%) were aged $75-90$ and 41656 (38.2\%) were younger than 60. The mean age for men was 63.9 (SD 9.4) and for women was 63.6 (SD 9.8); 49.4\% were women (table 1). About one third of participants were in or had been in a low occupational class; $35.4 \%$ of men and $24.8 \%$ of women were in or had been in a high occupational class.

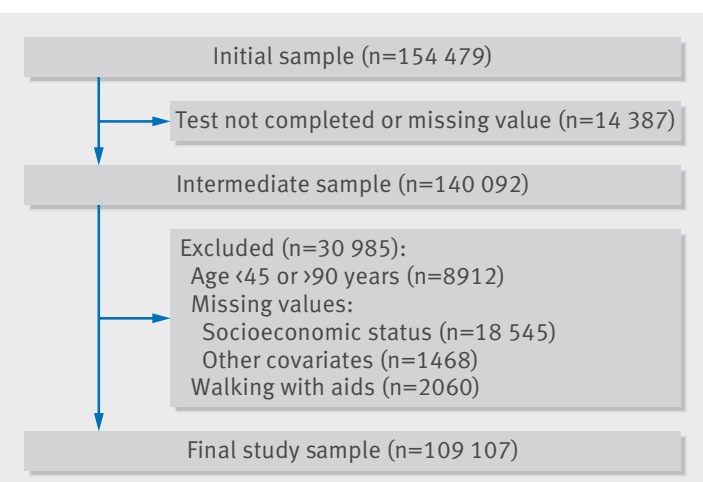

Fig 1 | Flow diagram of participants included in study 


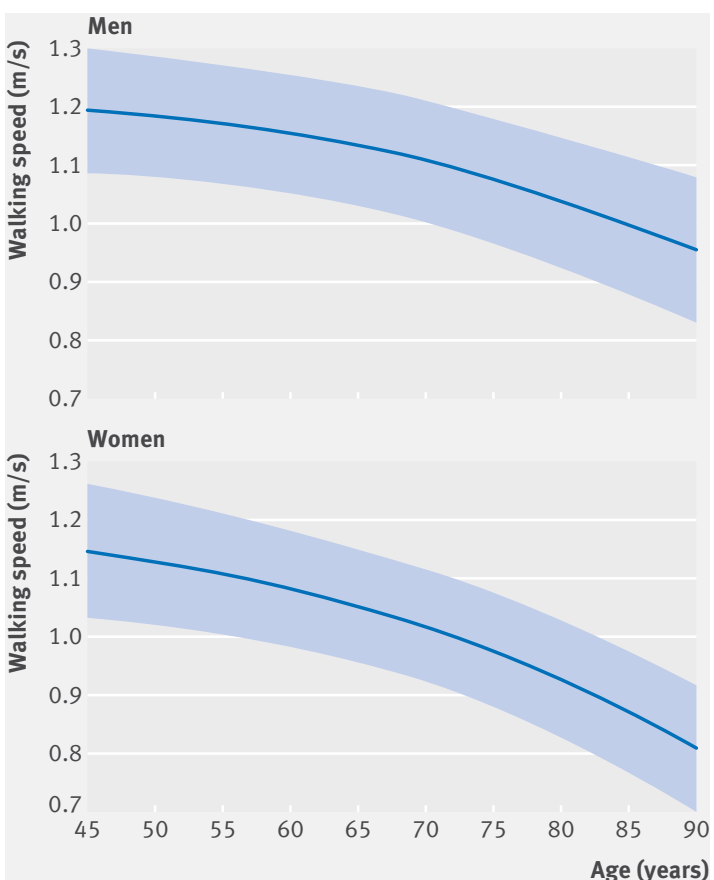

Fig 2 | Walking speed as function of age in men and women

Figure 2 shows the age related decline in walking speed in 55255 men and 53852 women predicted using the GAMM model, conditioned to the average height of men and women. For men, walking speed declined from $1.19 \mathrm{~m} / \mathrm{s}$ (95\% confidence interval 1.08 to 1.30$)$ at age 45 to $0.95 \mathrm{~m} / \mathrm{s}$ (0.83 to 1.08$)$ at age 90 . For women, the decline was from $1.15 \mathrm{~m} / \mathrm{s}$ (1.03 to $1.26)$ at age 45 to $0.81 \mathrm{~m} / \mathrm{s}(0.70$ to 0.92$)$ at age 90 . Age related decline in walking speed was not linear but accelerated after the ages of $65-70$ for both men and women.

\section{Years of functioning lost by age 60 and 85}

Figure 3 shows the years of functioning lost by age 60 due to exposure to suboptimal risk factors, as measured by differences in walking speed. A 60 year old man of low socioeconomic status had the same walking speed as a 66.6 year old man of high socioeconomic status (95\% confidence interval 5.0 to 9.4 ), whereas for women the difference was 4.6 (3.6 to 6.2) years. Years of functioning lost due to low socioeconomic status were comparable to years of functioning lost due to obesity, diabetes, and physical inactivity (range 5.1 to 5.6 for men and 5.4 to 7.5 for women), but they were greater than years lost due to tobacco smoking, hypertension, and high alcohol intake (range 0.7 to 3.0 for men and 3.0 to -0.1 for women). Results were comparable in analyses mutually controlling for all risk factors. Analyses were repeated using a two step approach including cohort specific analyses and pooling of cohort specific estimates using meta-analysis (see supplementary appendix 4). The approach yielded results similar to those found in the main analysis (see figure 3). Finally, we also estimated years of functioning lost by ages 50

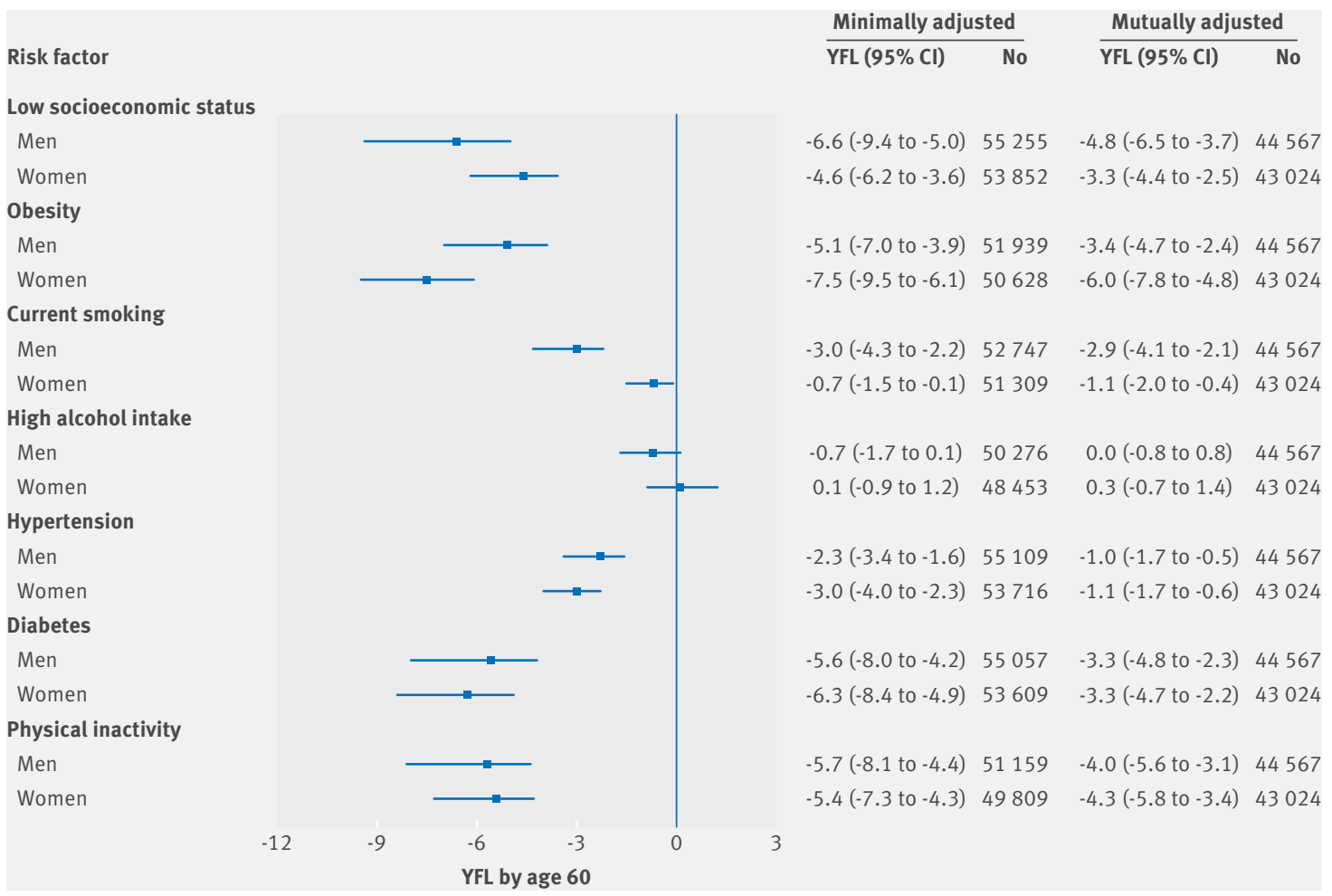

Fig 3 | Years of functioning lost (YFL) by age 60 due to suboptimal risk factors. Minimally adjusted models were only adjusted for age, age $^{2}$, height, year of birth, and distances walked; in mutually adjusted models, socioeconomic status and non-communicable diseases risk factors are mutually adjusted 
and 70 (see supplementary appendix 3, figures S2 and S3); the pattern and magnitude of the associations between risk factor and years of functioning lost were comparable to those observed by age 60 .

In analyses restricted to high income countries, years of functioning lost attributable to low socioeconomic status by age 60 were 8.0 (95\% confidence interval 5.7 to 13.1) for men and 5.4 (4.0 to 8.0) for women (see supplementary appendix 3, figures S4 and S5). In low and middle income countries, the corresponding years of functioning lost were 2.6 ( 0.2 to 6.8 ) for men and 2.7 (1.0 to 5.5) for women. Within high income countries, years of functioning lost attributable to low socioeconomic status by age 60 were greater in the United States than in Europe (see supplementary appendix 3, figures S6 and S7): 19.7 (13.2 to 27.4) $v$ 6.0 (3.8 to 12.1 ) for men and 15.8 (10.8 to 21.4$) v 3.9$ (2.6 to 7.2) for women. This was also the case for years of functioning lost attributable to other risk factors, in particular current smoking and high alcohol intake.

In a sensitivity analysis including participants who used walking aids, the number of years of functioning lost attributable to risk factors by age 60 did not materially differ from those reported in the main analysis (see supplementary appendix 3, figure S8). In supplementary multivariate analyses, adjusting for physical inactivity, ethnicity, or health status (see supplementary appendix 3, table S2-S4), or for the number of other risk factors to which participants were exposed (see supplementary appendix 3, table S6), years of functioning lost attributable to the risk factors by age 60 did not differ from those reported in the main analysis.

In an additional analysis where the maximum number of participants for each risk factor was used irrespective of the availability of socioeconomic status data (see supplementary appendix 3, table S5), the estimated years of functioning lost attributable to the non-communicable disease risk factors by age $60 \mathrm{did}$ not differ from those reported in the main analysis. The association between obesity and walking speed was little changed at age 60 after inclusion of underweight participants in the reference group (see supplementary appendix 3, table S7).

Figure 4 shows years of functioning lost due to suboptimal risk factors by age 85 . Walking speed continued to decrease as a function of risk factors between ages 60 and 85 (see supplementary appendix 3 , figure S1), in particular for those of low versus high socioeconomic status (11.1 (95\% confidence interval 7.1 to 15.7 ) years of functioning lost at age 85 for men and 6.7 (4.5 to 11.2) for women) and for those with insufficient versus sufficient physical activity (16.7 (10.5 to 25.8) years of functioning lost at age 85 for men and 16.3 (10.7 to 24.8) for women).

\section{Years of life lost between ages 60 and 85}

Years of functioning lost were greater than years of life lost due to low socioeconomic status and noncommunicable disease risk factors. Between ages 60 and 85 years, low socioeconomic status was associated with a loss of 0.6 (95\% confidence interval 0.4 to 0.9 )

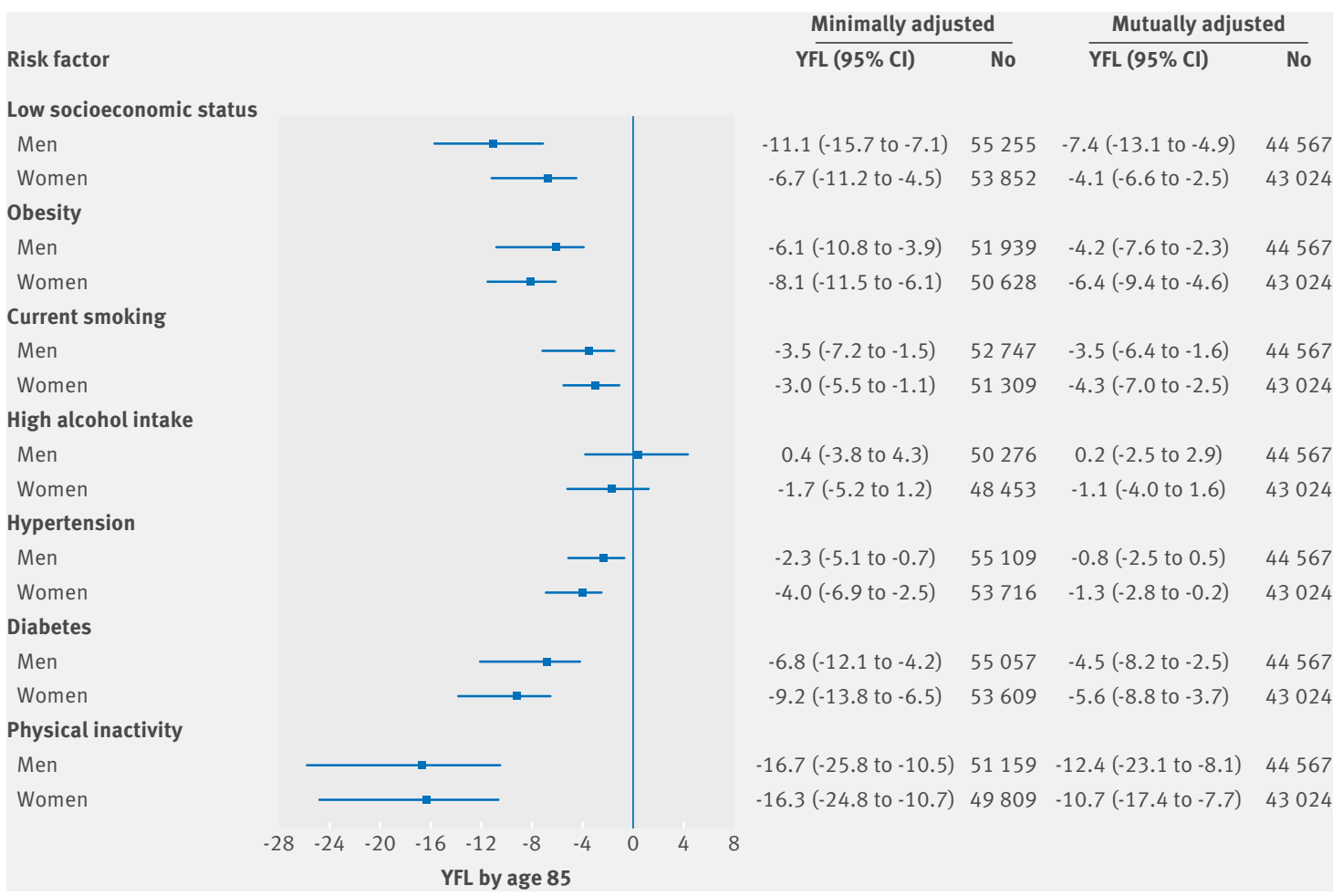

Fig 4 | Years of functioning lost (YFL) by age 85 due to suboptimal risk factors. Minimally adjusted models were only adjusted for age, age $^{2}$, height, year of birth, and distances walked; in mutually adjusted models, socioeconomic status and non-communicable diseases risk factors are mutually adjusted 


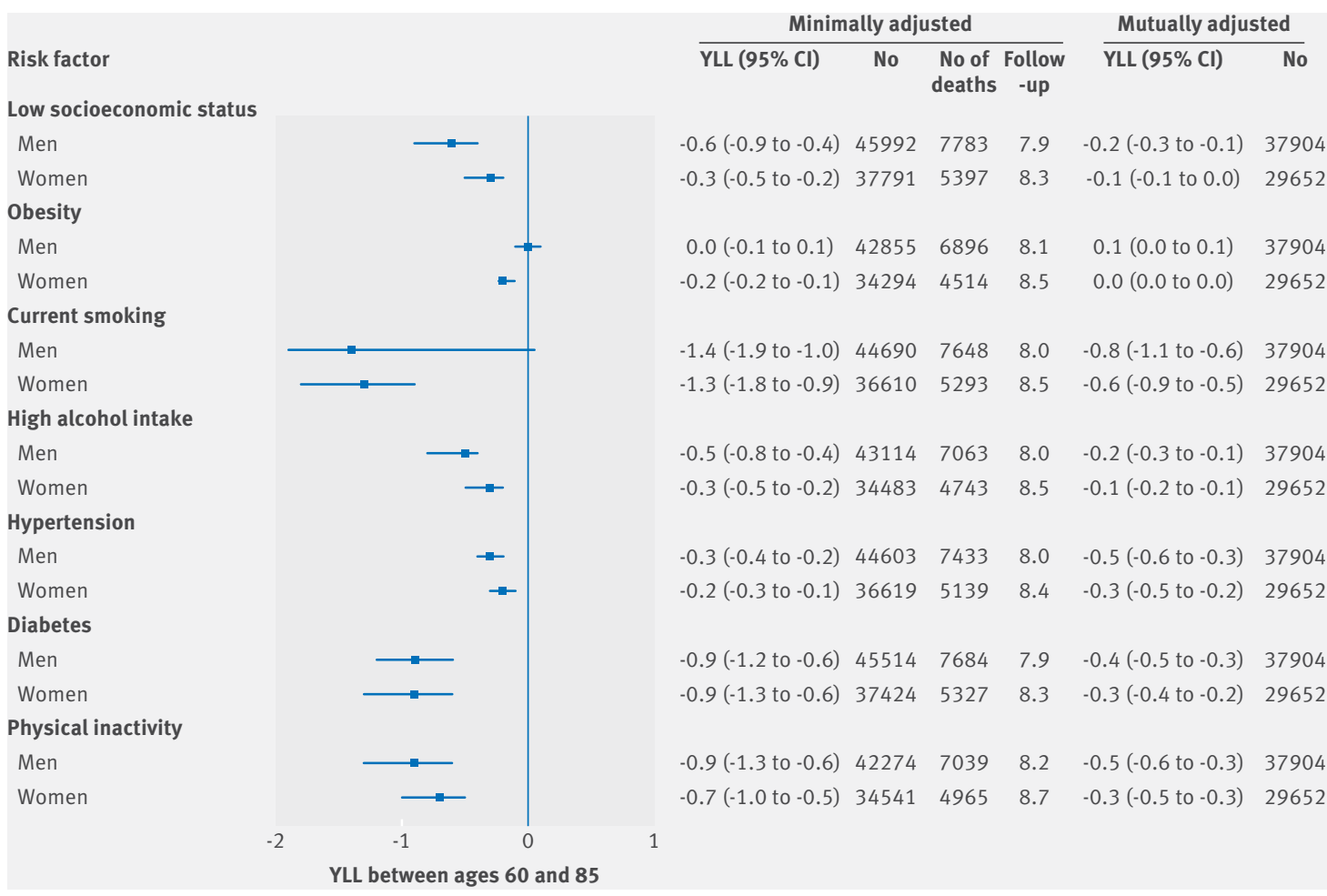

Fig 5 | Years of life lost (YLL) between ages 60 and 85 due to suboptimal risk factors. Minimally adjusted models were only adjusted for year of birth; in mutually adjusted models, socioeconomic status and non-communicable diseases risk factors are mutually adjusted

years of life for men and 0.3 (0.2 to 0.5$)$ years of life for women (fig 5). Years of life lost due to suboptimal risk factors were similar for high alcohol intake ( 0.5 for men and 0.3 for women), greater for smoking (1.4 for men and 1.3 for women), diabetes (0.9), and physical inactivity ( 0.9 for men and 0.7 for women), and lower for hypertension and obesity.

\section{Discussion}

Based on individual level data from 37 cohort studies and more than 109000 adults, our analyses showed that adverse socioeconomic circumstances and exposure to major non-communicable disease risk factors are robustly associated with loss in physical functioning from early to a more advanced old age. By age 60 , about six years of good physical functioning were lost due to poor socioeconomic circumstances. The years of functioning lost were comparable (ie, 5-7 years) for obesity, diabetes, and insufficient physical activity, and lower (between $<1$ and 3 years) for tobacco smoking, hypertension, and high alcohol intake. Exposure to adverse socioeconomic circumstances and the non-communicable disease risk factors continued to be associated with physical functioning after age 60, with increasing differences in functioning between exposed and unexposed groups at age 85 . Years of functioning lost at older ages generally exceeded the years of life lost, suggesting that an exclusive focus on morbidity and mortality might lead to underestimation of the potential benefits of targeting poor socioeconomic circumstances and risk factors highlighted in global health strategies. The years of functioning lost due to these factors were particularly large in high income countries.

\section{Strengths and weaknesses of this study}

We used walking speed to assess physical functional capacity. Previous research has shown that walking speed declines with age and is an independent predictor of survival, functional limitations, hospital admission, and cognitive decline. ${ }^{19} 2223$ Compared with other measures of physical functioning, such as grip strength, walking speed requires the coordinated action of several different physical systems, including the nervous, musculoskeletal, and cardiopulmonary systems. ${ }^{37}$ Furthermore, walking speed is widely used in aging research as it is easily measurable in population surveys as well as in clinical settings. However, heterogeneity did exist in the measurement of walking speed between studies, which we partially accounted for through a mixed statistical modelling framework. Since walking speed is unlikely to capture all important aspects of "overall functional capacity," further research with other indicators of physical functioning, such as grip strength and lung function would provide a useful comparison.

As our analyses relied on cross sectional data, these findings should be interpreted cautiously and should not be considered as causal estimates of the impact of socioeconomic status on health. For example, selection bias would attenuate observed associations if nonparticipation in studies was greater among those with 
poor functioning. Similarly, physical inactivity and obesity may be both a cause and a consequence of impaired mobility, potentially inflating the observed associations. However, our findings highlight the potential importance of socioeconomic status for physical functioning at older ages, and motivate further research to establish the causal nature of the observed associations. Randomised trials and quasiexperimental studies are required to evaluate the extent to which intervening on socioeconomic adversity and standard non-communicable disease risk factors might improve functional capacity in old age.

Occupational class was used as an indicator of socioeconomic status, as there were data available for this measure across the cohorts included in our study and occupational class was comparable between countries. Occupational class may not entirely reflect current socioeconomic conditions, particularly for older participants, although pensions and benefits after retirement are often a function of employment during active life. Since we compared the associations of low socioeconomic status with those of major noncommunicable disease risk factors that are currently targeted by global health strategies, we did not include additional factors that might also affect functional capacity, such as psychosocial stress, social isolation, and exposure to environmental pollutants; the effects of these factors are an important topic of further studies.

Socioeconomic status and non-communicable disease risk factors were assessed using broad categorisations, and for some of the cohort studies only self reported data were available. Furthermore, the success in harmonisation varied between cohort studies depending on the availability of data. As a result misclassifications might exist, potentially underestimating or overestimating the associations between socioeconomic status, non-communicable disease risk factors, and physical functioning.

\section{Comparison with previous studies}

Our study shows that the number of years of functioning lost due to adverse socioeconomic circumstances is comparable to or larger than the number of years lost due to major risk factors for chronic diseases, such as insufficient physical activity, diabetes, obesity, and tobacco consumption. Interestingly, this pattern persisted in models that controlled for all risk factors simultaneously. Risk factors, including socioeconomic adversity, tend to cluster in the same individuals, but our findings suggest that the association of low socioeconomic status with physical functioning is not attributable to non-communicable disease risk factors.

Years of functioning lost due to low socioeconomic status were greater in high income countries than in low to middle income countries. A potential explanation of this finding includes regional differences in the social patterning of major risk factors, such as physical inactivity, obesity, and diabetes. Unlike in high income countries, lower socioeconomic status is not always associated with a higher prevalence of these risk factors in low to middle income countries. ${ }^{38} 39$ In addition, classifying individuals into occupational classes in low to middle income countries is difficult as large fractions of the population have informal jobs or employment contracts, and misclassification of occupational class may have attenuated the observed associations. Furthermore, life expectancy is shorter in low to middle income countries and this may lead to greater selection bias attenuating associations in studies from these countries.

Insufficient physical activity, obesity, and diabetes were strong independent predictors of loss of functional capacity (5-7 years of functioning lost), whereas the association of tobacco consumption, high alcohol intake, and hypertension with loss of functional capacity was weaker (0-3 years of functioning lost). The result was expected, given the adverse impact of insufficient physical activity, obesity, and diabetes on the musculoskeletal system. Our findings are consistent with studies showing that physical activity might improve physical performance ${ }^{1540}$ even at older ages, ${ }^{41-43}$ although part of the observed effect is also likely to be due to a reverse causation process whereby decreasing mobility induces reductions in physical activity. Our study confirms the existing literature linking obesity and diabetes to walking performances, the plausible underlying mechanisms including the excess risk of osteoarthritis in the legs ${ }^{44}$ and lower extremity function in people aged 45-90 with obesity and diabetes. ${ }^{45}$

\section{Meaning of the study}

Our findings suggest that policies to deal with poor socioeconomic circumstances, in addition to common non-communicable disease risk factors, might be critical to strategies for the promotion of healthy aging. As such, this study supports the hypothesis that a successful implementation of the WHO Global Action Plan for the Prevention and Control of NonCommunicable Diseases and policies to reduce socioeconomic adversity might benefit physical functioning of the population. As with standard non-communicable diseases risk factors, the social environment is modifiable by policies at the local, national, and international levels. ${ }^{46}$ Examples of potential interventions include promoting early childhood development, tackling poverty and living circumstances, ensuring that all children have access to high quality education, and creating safe school and work environments by legislation. ${ }^{48} 49$ Given that the present study is based on observational data, our study informs about associations but cannot provide evidence of causality. Further research is needed to determine whether interventions targeting non-communicable disease risk factors and adverse socioeconomic circumstances early in life can potentially slow functional decline.

\section{Conclusions and implications for future research} Current global health policies are targeted towards established risk factors of health, such as smoking and 
physical inactivity. Much of the evidence of the benefits of reducing these factors involve hard endpoints, such as mortality and morbidity, whereas few studies have focused on additional intervention targets such as socioeconomic circumstances, or broader measures of wellbeing, such as physical functioning. Our findings from cohort studies in Europe, the United States, Latin America, Africa, and Asia address this limitation and show comparable associations of adverse socioeconomic circumstances and standard non-communicable disease risk factors with reduced walking speed, a measure of physical functioning from early old age onwards. Years of functioning lost were greater than years of life lost due to these risk factors. This evidence calls for interventional research on potential benefits of broader health policies dealing with socioeconomic adversity, in addition to standard risk factors.

\section{AUTHOR AFFILIATIONS}

${ }^{1}$ Institute of Social and Preventive Medicine, Lausanne University Hospital, Biopôle 2-Route de la Corniche 10, 1010 Switzerland

${ }^{2}$ Department of Psychology and Logopedics, Faculty of Medicine, University of Helsinki, Helsinki, Finland

${ }^{3}$ Department of Global Health and Social Medicine, King's College London, London, UK

${ }^{4}$ Harvard T.H. Chan School of Public Health, Boston MA, USA

${ }^{5}$ The Irish Longitudinal Study on Ageing (TILDA), Trinity College Dublin, Dublin, Ireland

${ }^{6}$ Epidemiology Unit, ASL TO3 Piedmont Region, Grugliasco (TO), Italy

${ }^{7}$ EPIUnit-Institute of Public Health, University of Porto, Porto, Portugal

${ }^{8}$ Department of Clinical Epidemiology, Predictive Medicine and Public Health, University of Porto Medical School, Porto, Portugal

${ }^{9}$ MRC-PHE Centre for Environment and Health, School of Public Health, Department of Epidemiology and Biostatistics, Imperial College London, London, UK

${ }^{10}$ INSERM, UMR1027, Toulouse, France, and Université Toulouse III Paul-Sabatier, Toulouse, France

${ }^{11}$ Population-based Epidemiological Cohorts Unit, INSERM UMS 11, Villejuif, France, and Paris Descartes University, Paris, France

${ }^{12}$ Cancer Epidemiology Centre, Cancer Council Victoria, Melbourne, VIC, Australia

${ }^{13}$ University College London, Department of Epidemiology and Public Health, London, UK

${ }^{14}$ Department of Sociology, Trinity College Dublin, Dublin, Ireland

${ }^{15} \mathrm{Global}$ Research Analytics for Population Health, Health Policy and Management, Columbia University, New York, NY, USA

${ }^{16}$ CESP, Inserm U1018, Université Paris-Saclay, Villejuif, France

${ }^{17}$ Human Genetics Foundation (HuGeF), Turin, Italy

${ }^{18}$ Department of Public Health, Erasmus University Medical Center, Rotterdam, Netherlands

${ }^{19}$ Clinicum, Faculty of Medicine, University of Helsinki, Finland

Members of the LIFEPATH Consortium (in alphabetical order): Jan Alberts, Harri Alenius, Mauricio Avendano, Laura Baglietto, Valeria Baltar, Mel Bartley, Henrique Barros, Michele Bellone, Eloise Berger, David Blane, Murielle Bochud, Giulia Candiani, Cristian Carmeli, Luca Carra, Raphaele Castagne, Marc Chadeau-Hyam, Sergio Cima, Giuseppe Costa, Emilie Courtin, Cyrille Delpierre, Angela Donkin, Angelo D’Errico, Pierre-Antoine Dugue, Paul Elliott, Guy Fagherazzi, Giovanni Fiorito, Silvia Fraga, Martina Gandini, Valérie Gares, Pascale Gerbouin-Rerolle, Graham Giles, Marcel Goldberg, Dario Greco, Allison Hodge, Michelle Kelly-Irving, Maryam Karimi, Piia Karisola, Mika Kivimaki, Jessica Laine, Thierry Lang, Audrey Laurent, Richard Layte, Benoit Lepage, Dori Lorsch, Giles Machell, Johan Mackenbach, Michael Marmot, Carlos de Mestral, Cathal McCrory, Cynthia Miller, Roger Milne, Peter Muennig, Wilma Nusselder, Dusan Petrovic.
Lourdes Pilapil, Silvia Polidoro, Martin Preisig, Ana Isabel Ribeiro, Fulvio Ricceri, Paolo Recalcati, Erica Reinhard, Oliver Robinson, Jose Rubio Valverde, Severine Saba, Frank Santegoets, Terrence Simmons, Gianluca Severi, Silvia Stringhini, Adam Tabak, Vesa Terhi, Joannie Tieulent, Salvatore Vaccarella, Federica Vigna-Taglianti, Paolo Vineis, Peter Vollenweider, Marie Zins.

Contributors: SS and CC contributed equally to the study. MKi and PV are joint last authors. MK, SS, and PV conceived the study. SS wrote the first and successive drafts of the manuscript. CC modelled and analysed the data. CC, MA, and MJ contributed to study conception and design. SS, MJ, CM, MG, and AIR contributed to data analysis. CC, MJ, MB, HB, GC, MG, GGG, RAK, MGM, AS, MJS, MZ, PV, and MK collected the data. All authors revised the manuscript for important intellectual content. SS and CC had full access to the data and take responsibility for the integrity of the data and the accuracy of the data analysis. SS is the guarantor.

Funding: This study was supported by the European Commission (Horizon 2020 grant No 633666) and the Swiss State Secretariat for Education, Research and Innovation SERI. SS was supported by an Ambizione grant (PZOOP3_167732) from the Swiss National Science Foundation. Silvia Fraga is supported by the Portuguese Foundation for Science and Technology (SFRH/BPD/97015/2013). Various sources have supported recruitment, follow-up, and measurements in the 48 cohort studies contributing to this collaborative analysis. MK is supported by the UK Medical Research Council (K013351), NordForsk, the Nordic Programme on Health and Welfare, the Academy of Finland (311492), and the Finnish Work Environment Fund. The funders had no role in the design and conduct of the study; collection, management, analysis, and interpretation of the data; and preparation, review, or approval of this manuscript.

Competing interests: All authors have completed the ICMJE uniform disclosure form at www.icmje.org/coi_disclosure.pdf and declare: no support from any organisation for the submitted work; no financial relationships with any organisations that might have an interest in the submitted work in the previous three years; no other relationships or activities that could appear to have influenced the submitted work.

Ethical approval: Not required.

Data sharing: No additional data available.

Transparency: The lead authors (SS and CC) affirm that his manuscript is an honest, accurate, and transparent account of the study being reported; that no important aspects of the study have been omitted; and that any discrepancies from the study as planned (and, if relevant, registered) have been explained.

This is an Open Access article distributed in accordance with the terms of the Creative Commons Attribution (CC BY 4.0) license, which permits others to distribute, remix, adapt and build upon this work, for commercial use, provided the original work is properly cited. See: http://creativecommons.org/licenses/by/4.0/.

1 Kontis V, Bennett JE, Mathers CD, Li G, Foreman K, Ezzati M. Future life expectancy in 35 industrialised countries: projections with a Bayesian model ensemble. Lancet 2017;389:1323-35. doi:10.1016/S0140-6736(16)32381-9

2 United Nations. World Population Prospects. In: United Nations, ed. Department of Economic and Social Affairs PD. 2015.

3 World Health Organization. Global action plan for the prevention and control of noncommunicable diseases 2013-2020. In: WHO, ed. Geneva, Switzerland, 2013.

4 Stringhini S, Carmeli C, Jokela M, et al, LIFEPATH consortium. Socioeconomic status and the $25 \times 25$ risk factors as determinants of premature mortality: a multicohort study and meta-analysis of 1.7 million men and women. Lancet 2017;389:1229-37. doi:10.1016/ S0140-6736(16)32380-7

5 Muennig P, Fiscella K, Tancredi D, Franks P. The relative health burden of selected social and behavioral risk factors in the United States: implications for policy. Am J Public Health 2010;100:1758-64. doi:10.2105/AJPH.2009.165019

6 Artaud F, Dugravot A, Sabia S, Singh-Manoux A, Tzourio C, Elbaz A. Unhealthy behaviours and disability in older adults: three-City Dijon cohort study. BMJ 2013:347:f4240. doi:10.1136/bmi.f4240

7 Bell JA, Sabia S, Singh-Manoux A, Hamer M, Kivimäki M. Healthy obesity and risk of accelerated functional decline and disability. Int J Obes (Lond) 2017:41:866-72. doi:10.1038/ijo.2017.51

8 Birnie K, Cooper R, Martin RM, et al, HALCyon study team. Childhood socioeconomic position and objectively measured physical capability levels in adulthood: a systematic review and meta-analysis. PLOS One 2011;6:e15564. doi:10.1371/journal.pone.0015564

9 Brunner E, Shipley M, Spencer V, et al. Social inequality in walking speed in early old age in the Whitehall II study. / Gerontol A Biol SC Med Sci 2009;64:1082-9. doi:10.1093/gerona/glp078 
10 Brunner EJ, Shipley MJ, Witte DR, et al. Arterial stiffness, physical function, and functional limitation: the Whitehal II Study. Hypertension 2011;57:1003-9. doi:10.1161/ HYPERTENSIONAHA.110.168864

11 Elbaz A, Shipley MJ, Nabi H, Brunner EJ, Kivimaki M, Singh-Manoux A Trajectories of the Framingham general cardiovascular risk profile in midlife and poor motor function later in life: the Whitehall II study. Int J Cardiol 2014;172:96-102. doi:10.1016/j.ijcard.2013.12.051

12 LaCroix AZ, Guralnik JM, Berkman LF, Wallace RB, Satterfield S. Maintaining mobility in late life. II. Smoking, alcohol consumption, physical activity, and body mass index. Am J Epidemiol 1993;137:858-69. doi:10.1093/oxfordjournals.aje. a116747

13 Lang IA, Llewellyn DJ, Alexander K, Melzer D. Obesity, physical function, and mortality in older adults. J Am Geriat Soc 2008:56:1474-8. doi:10.1111/j.1532-5415.2008.01813.x

14 Sabia S, Elbaz A, Rouveau N, Brunner EJ, Kivimaki M, Singh-Manoux A. Cumulative associations between midlife health behaviors and physical functioning in early old age: a 17-year prospective cohor study. J Am Geriatr Soc 2014;62:1860-8. doi:10.1111/jgs.13071

15 Stuck AE, Walthert JM, Nikolaus T, Büla CJ, Hohmann C, Beck JC. Risk factors for functional status decline in community-living elderly people: a systematic literature review. Soc Sci Med 1999;48:445-69. doi:10.1016/S0277-9536(98)00370-0

16 Zaninotto P, Sacker A, Head J. Relationship between wealth and age trajectories of walking speed among older adults: evidence from the English Longitudinal Study of Ageing. J Gerontol A Biol Sci Med Sci 2013;68:1525-31. doi:10.1093/gerona/glt058

17 McCrory C, Henretta JC, O'Connell MD, et al. Intergenerational Occupational Mobility and Objective Physical Functioning in Midlife and Older Ages. J Gerontol B Psychol Sci Soc Sci 2018;73:279-91. doi:10.1093/geronb/gbv084

18 Seeman T, Chen X. Risk and protective factors for physical functioning in older adults with and without chronic conditions: MacArthur Studies of Successful Aging. J Gerontol B Psychol Sci Soc Sci 2002;57:S135-44. doi:10.1093/geronb/57.3.S135

19 Studenski S, Perera S, Patel K, et al. Gait speed and survival in older adults. JAMA 2011;305:50-8. doi:10.1001/jama.2010.1923

20 Clark DJ, Rose DK, Ring SA, Porges EC. Utilization of central nervous system resources for preparation and performance of complex walking tasks in older adults. Front Aging Neurosci 2014;6:217. doi:10.3389/fnagi.2014.00217

21 Middleton A, Fritz SL, Lusardi M. Walking speed: the functional vital sign. J Aging Phys Act 2015;23:314-22. doi:10.1123/japa.20130236

22 Cesari M, Kritchevsky SB, Penninx BW, et al. Prognostic value of usual gait speed in well-functioning older people--results from the Health, Aging and Body Composition Study. J Am Geriatr Soc 2005; 53: 1675-80 doi:10.1111/j.1532-5415.2005.53501.x

23 Buracchio T, Dodge HH, Howieson D, Wasserman D, Kaye J. The trajectory of gait speed preceding mild cognitive impairment. Arch Neurol 2010;67:980-6. doi:10.1001/archneurol.2010.159

24 Cesari M, Onder G, Russo A, et al. Comorbidity and physical function: results from the aging and longevity study in the Sirente geographic area (ilSIRENTE study). Gerontology 2006;52:24-32. 10.1159/000089822

25 Cesari M, Penninx BW, Pahor M, et al. Inflammatory markers and physical performance in older persons: the InCHIANTI study. J Gerontol A Biol Sci Med Sci 2004;59:242-8. doi:10.1093/ gerona/59.3.M242

26 Abellan van Kan G, Rolland Y, Andrieu S, et al. Gait speed at usual pace as a predictor of adverse outcomes in community-dwelling older people an International Academy on Nutrition and Aging (IANA) Task Force. J Nutr Health Aging 2009;13:881-9. doi:10.1007/ s12603-009-0246-z

27 Elbaz A, Ripert M, Tavernier B, et al. Common carotid artery intima-media thickness, carotid plaques, and walking speed. Stroke 2005;36:2198-202. doi:10.1161/01. STR.0000181752.16915.5c

28 Odden MC, Peralta CA, Haan MN, Covinsky KE. Rethinking the association of high blood pressure with mortality in elderly adults: the impact of frailty. Arch Intern Med 2012;172:1162-8. doi:10.1001/archinternmed.2012.2555

29 Elbaz A, Sabia S, Brunner E, et al. Association of walking speed in late midlife with mortality: results from the Whitehall II cohort study. Age (Dordr) 2013;35:943-52. doi:10.1007/s11357-012-9387-9
30 Vineis P, Avendano-Pabon M, Barros H, et al. The biology of inequalities in health: the LIFEPATH project. Longitudinal and Life Course Studies, [S.I.], v. 8, n. 4, p. 417-449, Oct. 2017. ISSN 17579597. www.llcsjournal.org/index.php/llcs/article/view/448

31 d'Errico A, Ricceri F, Stringhini S, et al, LIFEPATH Consortium. Socioeconomic indicators in epidemiologic research: A practical example from the LIFEPATH study. PLoS One 2017;12:e0178071. doi:10.1371/journal.pone.0178071

32 Institute for Social and Economic Research. The European Socioeconomic Classification. www.iser.essex.ac.uk/archives/esec/userguide/the-european-socio-economic-classification.

33 Rose D, Harrison E. THE EUROPEAN SOCIO-ECONOMIC CLASSIFICATION: A NEW SOCIAL CLASS SCHEMA FOR COMPARATIVE EUROPEAN RESEARCH. Eur SOC 2007;9:459-90. doi:10.1080/ 14616690701336518

34 Wang Y. Mixed effects smoothing spline analysis of variance. I R Stat Soc Series B Stat Methodol 1998;60:159-74. doi:10.1111/14679868.00115

35 Wood SN. Stable and efficient multiple smoothing parameter estimation for generalized additive models. I Am Stat Assoc 2004;99:673-86. doi:10.1198/016214504000000980.

36 Morris JS. The BLUPS are not "best" when it comes to bootstrapping. Stat Probab Lett 2002:56:425-30 doi:10.1016/S0167. 7152(02)00041-X.

37 Hornyak V, VanSwearingen JM, Brach JS. Measurement of Gait Speed. Top Geriatr Rehabil 2012;28:27-32. doi:10.1097/ TGR.0b013e318233e75b.

38 Stringhini S, Viswanathan B, Gédéon J, Paccaud F, Bovet P. The social transition of risk factors for cardiovascular disease in the African region: evidence from three cross-sectional surveys in the Seychelles. Int J Cardiol 2013;168:1201-6. doi:10.1016/j.ijcard.2012.11.064

39 Hosseinpoor AR, Bergen N, Kunst A, et al. Socioeconomic inequalities in risk factors for non communicable diseases in low-income and middle-income countries: results from the World Health Survey. BMC Public Health 2012;12:912. doi:10.1186/1471-2458-12-912

40 Cooper R, Mishra GD, Kuh D. Physical activity across adulthood and physical performance in midlife: findings from a British birth cohort. Am J Prev Med 2011;41:376-84. doi:10.1016/j. amepre.2011.06.035

41 Bendall MJ, Bassey EJ, Pearson MB. Factors affecting walking speed of elderly people. Age Ageing 1989;18:327-32. doi:10.1093/ ageing/18.5.327

42 Pahor M, Guralnik JM, Ambrosius WT, et al, LIFE study investigators. Effect of structured physical activity on prevention of major mobility disability in older adults: the LIFE study randomized clinical trial. IAMA 2014:311:2387-96 doi:10.1001/jama.2014.5616

43 Stenholm S, Koster A, Valkeinen $\mathrm{H}$, et al. Association of Physical Activity History With Physical Function and Mortality in Old Age. | Gerontol A Biol Sci Med Sci 2016;71:496-501. doi:10.1093/ gerona/glv111

44 Ko S, Stenholm S, Ferrucci L. Characteristic gait patterns in older adults with obesity--results from the Baltimore Longitudinal Study of Aging. / Biomech 2010;43:1104-10. doi:10.1016/i. ibiomech.2009.12.004

45 Chiles NS, Phillips CL, Volpato S, et al. Diabetes, peripheral neuropathy, and lower-extremity function. J Diabetes Complications 2014;28:91-5. doi:10.1016/j.jdiacomp.2013.08.007

46 Commission for the Social Determinants of Health. Closing the gap in a generation: health equity through action on the social determinants of health. Final Report of the Commission on Social Determinants of Health. World Health Organization, 2008.

47 Marmot MG, Atkinson T, Bell J, et al. Fair society, healthy lives: A strategic review of health inequalities in England post-2010: The Marmot Review, 2010

48 Lleras-Muney A. The Relationship Between Education and Adult Mortality in the United States. Rev Econ Stud 2005;72:189-221. doi:10.1111/0034-6527.00329.

49 Heckman JJ. Skill formation and the economics of investing in disadvantaged children. Science 2006;312:1900-2. doi:10.1126/ science. 1128898

Supplementary information: descriptions of included cohort studies, tables S1-S7, figures S1-S8, and sensitivity analyses using a two step approach 